Para citar este artículo

Saldarriaga, C. y Patiño, B. (2016). Transferencia de valores económicos para la estimación de impactos sobre cobertura boscosa en proyectos hidroeléctricos. Económicas CUC, 37(1), 41-60. DOI: http://dx.doi. org/10.17981/econcuc.37.1.2016.02

\title{
Transferencia de valores económicos para la estimación de impactos sobre cobertura boscosa en proyectos hidroeléctricos
}

DOI: http://dx.doi.org/10.17981/econcuc.37.1.2016.02

\author{
Carlos Saldarriaga ${ }^{1}$ \\ Bernardo Patiño ${ }^{2}$
}

\begin{abstract}
Resumen
En este artículo se realiza una revisión del método de transferencia de beneficios, con una aplicación particular al establecimiento del valor económico asociado a los impactos sobre cobertura boscosa en un proyecto hidroeléctrico ubicado en el departamento de Antioquia Colombia. Los resultados del estudio, indican que los valores económicos del impacto en bosque son más confiables cuando se obtienen a partir la percepción de los hogares, a través de su disposición a pagar por conservación o pérdida de bienestar. Esto último se logra por medio de la aplicación de métodos de preferencias declaradas o de preferencias reveladas. Se estima, que en presencia de limitaciones de tiempo y de disponibilidad de recursos, las agencias gubernamentales pueden tomar decisiones de política pública, con relación a priorización de recursos, a partir de la realización de análisis costo-beneficio basados en el método de transferencia de beneficios.
\end{abstract}

Palabras clave: valoración económica de impactos ambientales; bosques; transferencia de beneficios; proyectos hidroeléctricos.

Recibido: 19.11.2015 Devuelto para revisión: 17.12.2015 Aceptado: 25.1.2016

\begin{abstract}
1 Doctor en Ingeniería de Sistemas; Universidad Nacional de Colombia- Medellín, Colombia. Magister en Economía Ambiental y de los Recursos Naturales; Universidad de Concepción-Chile. Economista; Universidad Nacional de ColombiaMedellín, Colombia. Profesor asistente e investigador adscrito al grupo Grupo Ciencias de la Decisión; Universidad Nacional de Colombia- Medellín, Colombia. Autor de correspondencia, casaldarriagai@unal.edu.co

${ }^{2}$ Maestrante en Ciencias económicas; Universidad Nacional de Colombia - Medellín, Colombia. Economista; Universidad de Medellín (UDEM) - Medellín, Colombia. Profesor de cátedra, consultor adscrito al grupo de investigación en economía aplicada (GEA); Universidad de Medellín (UDEM) - Medellín, Colombia. berand13@gmail.com
\end{abstract}

- The author; licensee Universidad de la Costa - CUC.

Económicas CUC vol. 37 no. 1, pp. 41-60. Enero - Junio, 2016 Barranquilla. ISSN 0120-3932 Impreso, ISSN 2382-3860 Online 


\title{
Transfer of Economic Values for the Estimation of Impacts on Forest Cover in Hydroelectric Projects
}

\begin{abstract}
This article submit a review of the benefits transfer method, with a specific application of the economic value establishment associated to the impacts on forest cover in a hydroelectric project located in the department of Antioquia - Colombia. The results indicated that economic values of forest impact are more reliable when obtained from the perception of households, through their willingness to pay for conservation or loss of welfare. This is achieved through the application of stated preference methods or revealed preferences. Is estimated that, with time and resource availability constraints, government agencies can make public policy decisions, in relation to resource prioritization, considering cost-benefit analysis based on the transfer benefits method.
\end{abstract}

Keywords: economic valuation of environmental impacts; forests; transfer benefits; hydroelectric; projects.

\section{Introducción}

Los efectos ambientales asociados al desarrollo de las actividades económicas de sectores productivos como el sector de generación de energía deterioran la calidad de vida de las personas que se ven expuestas a cambios en sus entornos (Hoffmann, 1996). Así, estos cambios en el bienestar de la población son parte importante de los problemas a considerar dentro de los análisis económicos actuales.
El análisis de los problemas ambientales desde el punto de vista económico es un reto latente dentro del desarrollo de la teoría económica (Azqueta, 1994) por su complejidad y por las altas necesidades de información que se requiere para el estudio de estos problemas, información que en algunos casos es escasa. Al momento de tomar decisiones sobre el uso de los recursos, el análisis de los costos y los beneficios asociados a la política se ve limitado por la dificultad de establecer el valor de los bienes 
ambientales que pueden ser potencialmente impactados con la decisión de política (Hoffmann, 1996). El valor económico de los recursos naturales y los ecosistemas, su contribución al progreso, su importancia en relación con el capital físico y humano, el grado de su agotamiento y deterioro, y los efectos de ese deterioro en el bienestar de las personas, son desconocidos en los inicios de los análisis económicos a desarrollar (Mendieta, 1999).

Particularmente, la ejecución de una adecuada valoración económica de los beneficios ambientales permite determinar que los ecosistemas, con un adecuado manejo, pueden usarse de manera más razonable y coherente con las necesidades futuras. Del mismo modo, la valoración de los costos ambientales, permite identificar los impactos negativos, producto de intervenciones inadecuadas de los recursos naturales.

Teniendo en cuenta lo anterior, llevar a cabo una correcta valoración de los impactos generados, en los bienes y servicios ambientales, a partir de los proyectos de generación de energía, es un punto de interés para la comunidad y para los diferentes agentes políticos y sociales (Guo, Li, Xiao, Zhang y Gan, 2007). Así, la valoración económica de los bienes y servicios de biodiversidad, implica la generación de criterios económicos ambientales para la toma de decisiones sobre el aprovechamiento de estos recursos. Además del tradicional análisis cualitativo, se ha introducido el concepto de análisis cuantitativo donde los beneficios y daños del aprovechamiento de estos recursos se expresan en términos monetarios (Azqueta, 1994).

Valoración significa valoración monetaria. El dinero se usa como patrón de medida para indicar las pérdidas o ganancias de utilidad o bienestar. La razón para utilizar el dinero como indicador a la hora de medir es que todos expresamos nuestras preferencias día a día en esos términos: al comprar bienes indicamos la "disposición a pagar" (DAP) intercambiando dinero por bienes y, a cambio, la DAP debe reflejar las preferencias. Como la valoración monetaria se relaciona con las preferencias individuales, el rechazo de cualquier preferencia, como la base adecuada para la toma de decisiones sobre el medio ambiente, implicaría rechazar los valores monetarios. "La valoración económica significa poder contar con un indicador de la importancia del medio ambiente en el bienestar social, y este indicador debe permitir compararlo con otros componentes del mismo" (Azqueta, 1994).

La riqueza ambiental es la base principal del desarrollo social y económico de 
un país. Ésta brinda cierto flujo de bienes y servicios ambientales que dependen del estado de los recursos naturales y del medio ambiente; es decir, el bienestar de la sociedad depende no sólo de los bienes y servicios generados por la actividad económica sino también de la calidad del medio ambiente.

Sin embargo, si bien se sabe intuitivamente que dichos recursos son importantes, esto tal vez no basta para garantizar su uso racional. Lo anterior se debe a que estos bienes y servicios ambientales carecen de un mercado donde intercambiarse $\mathrm{y}$, en consecuencia, se desconoce su precio. Así, la ausencia de la valoración de estos recursos puede llevar a la sobreexplotación o al uso inadecuado y, por tanto, a que dejen de generar los flujos de beneficios necesarios para mantener el bienestar social.

El caso de la generación de energía y su utilización para la ejecución de diferentes actividades económicas asociadas a la satisfacción de las necesidades de producción y consumo, ha constituido en un hilo conductor, desde el punto material, en la historia del progreso económico. En América Latina existe un uso permanente de la hidroelectricidad, a saber, pues las condiciones del territorio permiten el empleo de las pendientes y resaltos topográficos para la generación de energía eléctrica. No obstante, el aprovechamiento del recurso genera impactos ambientales importantes sobre la biodiversidad y las coberturas vegetales que aún proliferan en los países en vía de desarrollo.

Cabe resaltar que la generación de energía hidroeléctrica se ha constituido en una fuerte alternativa de consumo y producción de energía, dado su origen de una fuente renovable, de generación masiva y aprovechable con altos niveles de eficiencia, utilización versátil y de bajo costo (AEVAL - Agencia de Evaluación de las Políticas Públicas y la Calidad de los Servicios, 2013). Actualmente, la energía generada por medios hidráulicos tiene una alta participación en el suministro de energía, no sólo a nivel mundial sino también en el entorno latinoamericano y nacional. Para Colombia, la generación de energía eléctrica está básicamente dominada por la generación de energía hidráulica con una participación del $64 \%$ de la producción total, mientras el proceso de generación más cercano alcanza el 33\% (termoeléctricas) de participación del mercado (MinMinas, 2012).

Esta situación crea la necesidad de establecer una relación entre la producción de energía hidroeléctrica y la conservación de los ecosistemas y biodiversidad, en particular la conservación y mantenimiento de las coberturas boscosas (Guo et al., 2007). Ahora, los efectos de generación de energía par- 
ten desde el cambio del uso de suelo asociado al cambio de actividad productiva de la zona además de los impactos asociados a las zonas de inundaciones y demás obras de ingeniería que eliminan cobertura boscosa.

En Colombia, tanto las autoridades ambientales como el sector privado (promotoras de proyectos de desarrollo) se apoyan de los procesos de valoración económica de bienes y servicios ambientales con el objetivo de tomar decisiones con respecto al uso, asignación y disposición de los activos ambientales entre alternativas como la conservación y realización de determinado proyectos. Situación que contrasta con la baja disponibilidad de estudios que permitan identificar la línea base (estado actual) de activos ambientales dispuestos en el territorio y la valoración en términos económicos de estos activos o capital natural.

Para asumir esta baja disponibilidad de información, los agentes interesados desarrollan actividades como la construcción de inventarios de los bienes ambientales asociados a las áreas de influencia de los proyectos de desarrollo, contenidos en los denominados Diagnóstico Ambiental de Alternativas (DAA) y Estudios de Impacto Ambiental (EIA) que permiten aproximarse al acervo de capital natural vinculados a estas zonas de política mediante una caracterización del área de influencia del proyecto para los medios biótico, abiótico y social (ANLA - Autoridad Nacional de Licencias Ambientales, 2006). En un sentido similar, la elaboración de los EIA exige la estimación de valores económicos para los bienes ambientales potencialmente impactados por la generación del proyecto de infraestructura donde se consideran una serie de metodologías económicas para la estimación de estos valores asociados a los bienes y servicios ambientales (Ministerio de Ambiente, Vivienda y Desarrollo Territorial - [MAVDT], 2010). Estas metodologías se basan en precios de mercado, gastos anuales y potenciales, preferencias reveladas y preferencias declaradas, lo que enmarca de forma inherente altos requerimientos de información disponible. De no existir esta información, requiere la asignación de recursos y tiempo para el proceso de obtención de información, donde se asumen de manera explícita retrasos en la toma de decisiones sobre la utilización de los recursos ambientales disponibles.

A partir de considerar algunas de las dificultades asociadas a la realización de investigaciones que implican tiempo y dinero para la toma de decisiones de aprovechamiento de recursos naturales, es necesario analizar instrumentos, herramientas o métodos económicos ágiles y confiables a disposición de las autoridades ambientales 
y agentes privados con el objetivo de llevar a cabo decisiones de política con resultados costo-eficientes en términos privados y sociales (Pearce y Tuener, 1990). Así, se cuenta con diferentes métodos de valoración económica que permiten obtener un indicador en unidades monetarias asociadas al cambio en el bienestar social provocado por un determinado cambio en la calidad ambiental. Estas metodologías poseen diferentes requerimientos de información primaria y secundaria para su aplicación (Vásquez, Cerda y Orrego, 2007). Los requerimientos de información, en contraste con la información disponible, determinan qué metodología emplear para los casos particulares. Dentro de las diferentes metodologías económicas que se pueden emplear para la monetización de impactos ambientales está la transferencia de beneficios (Bennett, 2011). La transferencia de beneficios permite el empleo de los valores monetarios de bienes ambientales estimados en un contexto determinado para estimar los beneficios de un bien parecido, o bajo distinto contexto, del cual se desconoce su valor (Desvouges et al., 1992). Lo anterior permite el uso de resultados previos de otras investigaciones desarrolladas en situaciones similares; así se convierte en una alternativa atractiva con respecto a hacer nuevas investigaciones que implican tiempo y recursos, lo que agiliza la toma de decisiones.
El presente artículo busca discutir la factibilidad de implementar la transferencia de beneficios en la estimación de valores económicos de impactos ambientales asociados al desarrollo de proyectos de infraestructura. Para esta propuesta, se consideran los proyectos de generación de hidroelectricidad como un generador de impactos ambientales importantes en los sistemas físico-biótico o ecosistemas en Antioquia (Colombia), y asumiendo el impacto sobre la cobertura boscosa como elemento de interés por la connotación que tiene el recurso bosque como un importante componente del capital natural de los ecosistemas en Antioquia.

\section{Transferencia de beneficios}

Como método de valoración económica de cambios en la calidad ambiental, la transferencia de beneficios consiste en el traspaso del valor monetario de un bien ambiental (denominado sitio de estudio) a otro bien ambiental (denominado sitio de intervención) (Brouwer, 2000). Este método permite evaluar el impacto de políticas ambientales cuando no es posible aplicar técnicas de valoración directas debido a restricciones presupuestarias y a límites de tiempo. 
Las cifras derivadas de la transferencia de beneficios constituyen una primera aproximación valiosa para quienes toman las decisiones acerca de los beneficios o costos de adoptar una política o programa.

La debilidad de la transferencia de beneficios radica en la confiabilidad y la validez de sus aproximaciones. La calidad de las aproximaciones depende en una buena medida de la validez de los estudios base para realizar la transferencia de beneficios y en la metodología utilizada. Sus desventajas potenciales son cuatro: primero, la calidad de los estudios originales determina completamente la confiabilidad del resultado de la transferencia; segundo, la valoración de ciertos bienes ambientales cuenta con un número reducido de estudios, lo cual restringe el espectro de información; tercero, los estudios de valoración ambiental no están diseñados para realizar transferencia de beneficios, redundando en dificultades para transferir valores del sitio de estudio al sitio de intervención (Rosenberger y Loomis, 2000); por último, los estudios de transferencia de beneficios sólo se deben aplicar cuando la necesidad de precisión en las medidas de bienestar es baja (Navrud y Bergland, 2001).

\section{- Pasos para la transferencia de beneficios}

La validez de los estudios de transferencia de beneficios se basa en la calidad del conjunto de estudios existentes y en la similitud entre el sitio de estudio y el sitio de intervención. Para asegurarse de cumplir con dichos requisitos, la aplicación de un estudio de transferencia de beneficios debe cumplir con los siguientes cuatro pasos (Departamento de Agricultura de los Estados Unidos - USDA, 2001):

a. Identificar el recurso o servicio objeto de la valoración: el primer paso requiere la identificación del recurso natural o servicio a evaluar, y caracterizar la naturaleza del incremento o reducción de oferta ambiental (p. ej. incremento en el caudal de agua). Es recomendable enumerar todos los beneficios económicos del recurso o servicio y establecer el nivel de la oferta ambiental en el sitio de estudio y el sitio de intervención.

b. Identificar los potenciales estudios relevantes para el ejercicio: para llevar a cabo el segundo paso, es necesario realizar una extensa revisión de bibliografía. Ello significa identificar estudios que valoran recursos o servicios similares a aquellos del sitio de intervención. 
c. Evaluar la aplicabilidad de los estudios existentes: en el tercer paso se deben revisar cuidadosamente los estudios identificados para establecer si sus medidas de beneficios son transferibles al sitio de intervención. Con el fin de aplicar los resultados de los estudios existentes, es necesario que la naturaleza de los recursos o servicios evaluados en el sitio de estudio sean comparables con los recursos o servicios del sitio de intervención. Las siguientes cuatro características deben cumplirse en un ejercicio adecuado de transferencia de beneficios:

i. Los estudios base de la transferencia de beneficios deben ser de alta calidad, es decir es imprescindible que sus datos sean adecuados y que los métodos económicos, así como las técnicas empíricas, sean correctos.

ii. Las características del recurso o servicio valorado y de la población que lo valora deben ser similares en el sitio de estudio y el sitio de intervención. Algunas características que deben guardar similitud entre el sitio de estudio y el sitio de intervención, éstas son:

- Grupo de población beneficiado por el recurso o servicio;
- Sitios sustitutos;

- Área geográfica;

- Características socio-demográficas (p. ej. edad, ingreso y educación); y

- Calidad de los recursos o servicios ambientales.

iii.El tamaño del mercado, es decir la población beneficiada por la existencia del recurso, debe ser similar en el sitio de estudio y en el sitio de intervención.

iv. La asignación de los derechos de propiedad del recurso ambiental en el sitio de estudio y el sitio de intervención debe ser igual con el propósito de utilizar la misma medida de bienestar (p. ej. disponibilidad a aceptar o disponibilidad a pagar).

d. Llevar a cabo la transferencia de beneficios: en el último paso se calculan los beneficios con base en los métodos descritos.

Hay dos conjuntos de métodos para realizar transferencia de beneficios: la transferencia de valores y la transferencia de funciones. Los métodos de transferencia de valores adaptan una medida de bienestar de un sólo estudio o una medida estadística de un conjunto de estudios, tal como la media, al sitio de intervención. 
La transferencia de funciones implica la transferencia de una función de beneficio o de demanda de un sitio de estudio o una regresión de meta-análisis de un conjunto de estudios al sitio de intervención (Rosenberger y Loomis, 2000).

\section{Transferencia de valores}

La transferencia de valores estimados se desarrolla para transferir valores estimados de un sitio a otro. Este tipo de transferencia asume que el valor marginal para un individuo promedio en un sitio de estudio de un bien ambiental es similar al valor ambiental promedio para un individuo en un sitio de política. A partir de lo anterior, es posible transferir la disposición a pagar media (DAP) estimada del sitio de estudio al sitio de política (Navrud y Ready, 2007). La selección de este valor unitario puede estar basada en estimaciones de uno o pocos estudios de valoración considerados con características cercanas a las presentes en el sitio de política, o basados en valores promedio a la revisión de la literatura existente. Para el caso de la presente propuesta, la posibilidad de utilizar pocos estudios para la selección del valor potencialmente transferible o un promedio estimado a partir de la revisión de estudios, constituye un estrategia metodológica muy conveniente ante la presencia de incertidumbre de estudios con estimaciones de valores económicos de impactos para bosque con características muy similares al del sitio de estudio.

El problema inherente a la transferencia de valores unitarios está asociado a las diferencias en las características socioeconómicas (ingreso, educación, entre otras) en general de los individuos en el sitio de política versus a las de los individuos en el sitio de estudio. También, en la dificultad de definir las unidades en las que está el valor a transferir, el cual depende de las características y las condiciones espaciales del sitio de estudio, situación que se presenta de manera común al aplicar la trasferencia de valores económicos entre países. Comúnmente para mitigar esta limitación se desarrollan dos estrategias: (i) determinar la elasticidad de ingreso de la DAP para las unidades evaluadas y (ii) elegir una tasa de cambio que permita representar el valor estimado en la misma moneda (Walsh, Johnson y McKean, 1992).

En particular, se menciona que la transferencia de valores consta de dos métodos: (i) la transferencia de beneficios de un único estudio; y (ii) la transferencia de un valor promedio. La transferencia de los beneficios de un único estudio se basa en un sólo estudio relevante para el sitio de intervención. La transferencia de un valor promedio apro- 
Tabla 1

Pasos para transferencia de valores.

\begin{tabular}{lc}
\hline Transferencia de beneficios de un único estudio & Transferencia de un valor promedio \\
\hline - Identificar el recurso o servicios afectados por la & - Identificar el recurso o servicios afectados por \\
acción ambiental propuesta. & la acción ambiental propuesta. \\
- Realizar una revisión bibliográfica extensa. & - Realizar una revisión bibliográfica extensa. \\
- Evaluar la relevancia y aplicabilidad de los datos & datos provistos en los sitios de estudio. \\
provistos en los sitios de estudio. & - Calcular la media, mediana e intervalos de \\
- Seleccionar una medida de bienestar de uno de los & confianza del 95\% para los beneficios provistos \\
estudios analizados. & en los estudios relevantes. \\
- Transferir la medida de los beneficios y agregar la & - Agregar los beneficios para el total de la \\
medida para la población beneficiada. & población beneficiada. \\
\hline
\end{tabular}

Fuente: Rosenberger y Loomis (2000).

xima los beneficios del sitio de intervención con base en un conjunto de estudios relevantes y aplicables para el caso en cuestión y calcula la medida de los beneficios con un momento estadístico como la media o la mediana. Los pasos para realizar las dos metodologías se resumen en la Tabla 1.

\section{Transferencia de funciones}

Este procedimiento suele ser más atractivo que la trasferencia de valores unitarios, ya que permite usar más información sobre las diferencias entre el sitio de política y el sitio de estudio y el efecto en la población. El beneficio estimado relacionado con la transferencia de un sitio de estudio a un sitio de política puede ser estimado por diferentes metodologías, preferencias reveladas (RP), costo de viaje $(\mathrm{CV})$, precios hedónicos $(\mathrm{PH})$, y por preferencias declaradas, valoración contingente (VC), experimentos de elección (EE). Para algunas de estas metodologías, una función de beneficio estimada es:

$$
D A P i j=f(G j, H i)
$$

Donde $D A P i j$ es la disposición a pagar de la población $i$ para un bien ambiental en un sitio $j$; $G j$ son las características ambientales del bien en el sitio $j$; y $H i$ son las características de la población $i$. Alternativamente, la función de beneficio puede predecir la $D A P$ media para la población en el sitio $j$ basada en la medida agregada para $H i$.

Para implementar este procedimiento, se analizan los estudios existentes en la lite- 
ratura con estimaciones para los parámetros de la función de $D A P$. Se recolectan los datos para los grupos independientes de variables $G$ y $H$ en el sitio de política descritas en la ecuación (1), y se calcula la $D A P$ de la población en el sitio de política (Rosemberger y Loomis, 2000). Para el caso de la presente propuesta, la alternativa metodológica ya mencionada es viable en la medida que sea posible encontrar, mediante la pertinente revisión de literatura, uno o varios estudios que estimen de manera clara y discernible el impacto de la infraestructura hidroeléctrica sobre la cobertura boscosa. De manera posterior, se recolectarían los datos pertinentes sobre el sitio de política a partir de una revisión de información secundaria y análisis de bases de datos. Otra arista que se desprende de la posibilidad de realizar transferencia de funciones es el meta-análisis, en el cual se replica el procedimiento anteriormente descrito con respecto a la determinación de los parámetros estimados en los estudios, sin embargo, se procede a aplicar regresión sobre los parámetros determinados de una síntesis de estudios. Esta alternativa está igualmente sujeta a la disponibilidad de estudios sobre la temática específica.

Las aplicaciones del método de transferencia de beneficios para la valoración económica de bosques son reducidas, y para la valoración de impactos en bosques asociados a proyectos hidroeléctricos, aún más. Sin embargo, se han desarrollado algunos estudios de transferencia aplicados a bosques, entre los cuales se mencionan los siguientes estudios: para estimar el valor económico total (VET) de bosques en México (Adger, Brown, Cervigni y Moran, 1995) y Peace y Peace (2001) para estimar el VET de los servicios ecosistémicos del bosque a escala global. Macías (2010) emplea la transferencia de beneficios para valorar los impactos ambientales de una planta de energía eléctrica y para valorar los servicios ecosistémicos provistos por el Sistema Nacional de Áreas Protegidas en Chile; Figueroa (2010) empleó este mismo método. Los anteriores estudios mencionados permiten identificar la utilización del método de transferencia de beneficios para la valoración de bosques en diferentes contextos.

Ahora, como ya se mencionó, la transferencia de funciones se compone de dos métodos: (i) la transferencia de una función de demanda o de beneficios y (ii) la estimación de una regresión de meta-análisis. La transferencia de una función de demanda o de beneficio consiste en adaptar una función de demanda o de beneficio de un estudio relevante al sitio de intervención. Una regresión de meta-análisis se estima con las características cuantificables de los estudios y las medidas de beneficios de cada 
Tabla 2

Pasos para transferencia de funciones.

\begin{tabular}{lc}
\hline Transferencia de beneficios de un único estudio & Transferencia de un valor promedio \\
\hline & - Identificar el recurso o servicios afectados por \\
- Identificar el recurso o servicios afectados por la & la acción ambiental propuesta. \\
acción ambiental propuesta. & - Realizar una revisión bibliográfica extensa. \\
- Realizar una revisión bibliográfica extensa. & - Evaluar la relevancia y aplicabilidad de los \\
- Evaluar la relevancia y aplicabilidad de los datos & datos provistos en los sitios de estudio. \\
provistos en los sitios de estudio. & - Estimar una regresión de meta-análisis. \\
- Adaptar la función de demanda o de beneficios & - Adaptar la regresión de meta-análisis de \\
de acuerdo con las características del sitio de & acuerdo con las características del sitio de \\
intervención. & intervención. \\
- Agregar la medida para la población beneficiada. & - Agregar los beneficios para el total de la \\
& población beneficiada. \\
\hline
\end{tabular}

Fuente: Rosenberger y Loomis (2000).

estudio. El propósito de dicha regresión es establecer la influencia de factores metodológicos y de características de los sitios de estudio sobre las medidas de beneficio. La ecuación estimada se puede utilizar para aproximar un beneficio para el sitio de intervención. Los pasos necesarios para llevar a cabo los dos métodos se resumen en la Tabla 2.

\section{Test de viabilidad para transferencia de beneficios}

Diferentes estudios han estimado un test de viabilidad que permita contrastar la validez de las estimaciones obtenidas mediante el método de transferencia de beneficios, tanto para transferencia de valores unitarios como para transferencia de funciones (Loomis, 1992; Parsons y Kealy, 1994; Brouwer y Spaninks, 1999; Rosenberger y Loomis, 2000). Una estrategia metodológica factible de utilización para contrastar la validez de las estimaciones asume típicamente que el valor estimado en el estudio base o desarrollado en un sitio de estudio es observable, o el estudio al calcular dicho valor económico se aproxima al valor real del activo ambiental.

Por notación se plantea que $V^{p}$ es el valor del bien ambiental que es observado y $V_{p p}$ es el valor aproximado estimado por el estudio. En términos de notación, $s$ es para el sitio de estudio y para el sitio de política. En la aplicación de la transferencia de beneficios, $V_{s s}$ se usa para representar un 
valor estimado, el cual es usado para hacer referencia a un valor similar al valor real del activo ambiental. $V_{s s}$ es transferido a un diferente pero similar sitio $j$, donde sitio $j$ es el sitio de política y el valor se denota como $V_{p s}$. Así:

$$
V_{p s}=V_{p}+\delta_{p s}
$$

donde $\delta_{p s}$ es el error asociado con la transferencia de beneficios del sitio $i$ al sitio $j$.

El test empírico de convergencia de viabilidad permite calcular el porcentaje de diferencia entre $V_{p s}$ y $V_{p p}$ :

$$
\% \Delta V_{i j}=\frac{\left[\left(V_{p s}-V_{p p}\right)\right]}{V_{p p}} * 100
$$

donde $i \neq j$.

Al tener las ecuaciones (2) y (3), se concluye que la medida de convergencia de viabilidad es $\delta_{p s} / V_{p p}$. Ahora $\delta_{p s}$ contiene dos sucesos que pueden explicar el error en la transferencia: (i) diferencias en las características de un sitio de estudio a un sitio de política que se puede denotar como $\phi_{p s}$ y (ii) errores asociados a la estimación de $V^{p}$ mediante $V_{p p}$ (ci) (Woodward y Wui, 2001). La asunción de cualquiera de los dos errores típicos que explican la viabilidad de transferencia mediante este test se adhiere a las particularidades del ejercicio (Navrud y Ready, 2007).

\section{Transferencia de beneficios en el caso de proyectos hidroeléctricos}

En este artículo se presentan los resultados de la transferencia de beneficios aplicada a un sitio de política ubicado en el departamento de Antioquia, Colombia, puntualmente en los municipios de San Carlos, San Luis y Puerto Nare (con énfasis en las área rurales), los cuales se constituyen en área de influencia del proyecto de aprovechamiento hidroeléctrico Porvenir II. El objetivo de la transferencia es estimar valores de referencia que den razón del valor económico del impacto sobre la cobertura boscosa presente en la zona de influencia del proyecto de aprovechamiento hidroeléctrico ya mencionado.

Para la transferencia de beneficios fue necesaria la revisión de bases de datos en búsqueda de estudios que estimaran el valor económico de la pérdida de bosques por intervención de proyectos de aprovechamiento hidroeléctrico. Las bases de datos consultadas fueron: EVRI, Science Direct, Springer, Journal, EBSCO, Econlit, Jstor, entre otros. Como resultado de la búsqueda en bases de datos se identificaron 103 artículos de investigación relacionados con la temática, de los cuales 46 hacen referencia explicita a la valoración económica del recurso bosques bajo diferentes esce- 
narios, principalmente, conservación de la cobertura boscosa, impactos de infraestructuras sobre los valores del bosques. Se seleccionaron 20 artículos que evalúan el impacto de intervención sobre el bosque a partir de infraestructuras, de los cuales 5 se identificaron como adecuados por las características de los estudios y las zonas de aplicación, como de interés para la transferencia de valores.

Para la selección de los estudios de caso elegidos para la trasferencia se consideraron los siguientes criterios: i) medida de bienestar estimada (unidad marginal DAP hogar/mes); ii) aplicación de preferencias declaradas para la valoración económica del impacto; iii) formato de encuesta aplicada; iv) similitud con el sitio de política (tipo de intervención e impacto evaluado); v) modelo econométrico empleado para la estimación de los valores económicos; vi) zona de desarrollo de la intervención (el proyecto afecta una zona rural o urbana); y vii) área de afectación directa. A partir de estos criterios de decisión, se reitera la posibilidad de uso de 5 estudios para la transferencia de beneficios. Estos estudios se desarrollan en diferentes partes del mundo como: Chile, Sri Lanka, Corea, China y Colombia. Éstos se presentan en la Tabla 3.
Tabla 3

Estudios seleccionados para la transferencia de valores económicos.

\begin{tabular}{llc}
\hline \multicolumn{1}{c}{ Estudio } & País & $\begin{array}{c}\text { Periodo del } \\
\text { estudio }\end{array}$ \\
\hline Ponce et al (2011) & Chile & 2007 \\
$\begin{array}{l}\text { Gunawardena } \\
(2010)\end{array}$ & Sri Lanka & 2003 \\
$\begin{array}{l}\text { Han, Kwak y } \\
\text { Yoo (2008) }\end{array}$ & Corea & 2002 \\
$\begin{array}{l}\text { Tao, Yan y } \\
\text { Zhan (2012) } \\
\begin{array}{l}\text { Correa, Osorio y } \\
\text { Mendieta (2010) }\end{array}\end{array}$ & Colombia & 2010 \\
\hline
\end{tabular}

Fuente: Elaboración propia.

De los anteriores estudios, se identificaron las DAP estimadas como valor económico del impacto generado por el desarrollo de proyectos de aprovechamiento hidroeléctrico sobre el recurso bosque. El atributo del bosque que se evalúa fue principalmente la pérdida de belleza escénica o pérdida de paisaje derivada de la intervención del proyecto hidroeléctrico. Las principales estimaciones y datos relevantes de los estudios, se presentan en la Tabla 4. 
Tabla 4

DAP estimada por diferentes estudios.

\begin{tabular}{lcccccc}
\hline $\begin{array}{c}\text { País del } \\
\text { estudio }\end{array}$ & $\begin{array}{c}\text { Periodo del } \\
\text { estudio }\end{array}$ & $\begin{array}{c}\text { DAP media } \\
\text { hogar/mes }\end{array}$ & $\begin{array}{c}\text { Unidad } \\
\text { monetaria }\end{array}$ & Método** $^{*}$ & $\begin{array}{c}\text { Zona de } \\
\text { afectación } \\
(\mathbf{R} / \mathbf{U})^{* *}\end{array}$ & $\begin{array}{c}\text { No. de } \\
\text { encuestas }\end{array}$ \\
\hline Chile & 2007 & $\$ 4.997$ & Chile Pesos & PD & $\mathrm{R}$ & 651 \\
Sri Lanka & 2003 & $\$ 342$ & Rupias & PD & $\mathrm{R}$ & 229 \\
Korea & 2002 & $\$ 2.542$ & Won & PD & $\mathrm{R}$ & 805 \\
China & 2010 & $\$ 20$ & Yuan & PD & $\mathrm{R}$ & 200 \\
Colombia & 2010 & $\$ 3.527$ & Col pesos & PD & $\mathrm{R}$ & 250 \\
\hline
\end{tabular}

* Preferencias declaradas

${ }^{* *}$ Rural (R), Urbana (U)

Fuente: Elaboración propia.

A partir de los valores de DAP por hogar/mes estimados por los diferentes estudios considerados, se realiza la transferencia de valores al sitio de estudio, el cual parte de desarrollar un ajuste por paridad de poder adquisitivo para luego emplear la transferencia de medidas de tendencia central. Luego, a partir de las medidas de tendencia cen- tral estimadas, se calcula un intervalo de confianza para generar valores de transferencia en dos escenarios sobre la estimación de la DAP, para lo cual se usa el límite superior del intervalo y la subestimación de la DAP estimada mediante el uso del límite inferior. El ajuste por PPA se genera a partir de la siguiente ecuación:

$$
\begin{gathered}
\text { PPA2014 }=\left(\operatorname{valor} \frac{D A P}{\text { mes }}\right) *\left(\frac{I P C \text { pais en estudio } 2014}{I P C \text { del año estudio }}\right) *\left(\frac{I N B \text { percaita Colombia } 2014}{I N B \text { percapita pais en estdio } 2014}\right) \\
*\left(\frac{1}{\text { Tas de cambio euro } / \text { dolar }}\right) *\left(\text { Tasa de cambio col } \frac{\text { pesos }}{\text { dolar }}\right)
\end{gathered}
$$


Tabla 5

Datos requeridos para la estimación de la transferencia de DAP en PPA (2014).

\begin{tabular}{lccccccc}
\hline \multicolumn{1}{c}{ País } & $\begin{array}{c}\text { Periodo } \\
\text { del } \\
\text { estudio }\end{array}$ & $\begin{array}{c}\text { DAP } \\
\text { media } \\
\text { hogar/mes }\end{array}$ & $\begin{array}{c}\text { Unidad } \\
\text { monetaria }\end{array}$ & $\begin{array}{c}\text { IPC } \\
\mathbf{2 0 1 4}\end{array}$ & $\begin{array}{c}\text { IPC } \\
\text { año del } \\
\text { estudio }\end{array}$ & $\begin{array}{c}\text { INB PPA } \\
\text { (2014) } \\
\text { país de } \\
\text { estudio }\end{array}$ & $\begin{array}{c}\text { Tasa de } \\
\text { cambio en } \\
\text { dólares } \\
\text { (2014) }\end{array}$ \\
\hline Chile & 2007 & $\$ 4.997$ & Chile Pesos & 1,044 & 1,078 & 21,580 & 613,12 \\
Sri & 2003 & $\$ 342$ & Rupias & 1,033 & 1,063 & 10,270 & 131,58 \\
Lanka & 2002 & $\$ 2.542$ & Won & 1,013 & 1,028 & 34,620 & 900,09 \\
Corea & 2010 & $\$ 20$ & Yuan & 1,020 & 1,033 & 13,130 & 6,17 \\
China & 2010 & $\$ 3.527$ & Col pesos & 1,029 & 1,023 & 12,900 & 2392,92 \\
Colombia & 20 & & & & & \\
\hline
\end{tabular}

Fuente: Elaboración propia.

Los datos requeridos para la estimación se relacionan en la Tabla 5 .

Tabla 6

DAP de estudios seleccionados en PPA (2014).

\begin{tabular}{lc}
\hline \multicolumn{1}{c}{ Estudio } & $\begin{array}{c}\text { DAP media hogar/ } \\
\text { mes PPA }\end{array}$ \\
\hline Ponce et al (2011) & $\$ 11.288$ \\
Gunawardena (2010) & $\$ 7.581$ \\
Han, Kwak y Yoo (2008) & $\$ 2.481$ \\
Tao, Yan y Zhan (2012) & $\$ 7.459$ \\
$\begin{array}{l}\text { Correa, Osorio y } \\
\text { Mendieta (2010) }\end{array}$ & $\$ 3.548$ \\
\hline
\end{tabular}

Fuente: Elaboración propia.
Las estimaciones de DAP en PPA a 2014 se muestran en la Tabla 6.

A partir de los cálculos estimados y la información recolectada, se estiman las siguientes medidas de tendencia central empleando los datos de DAP estimados en paridad de poder adquisitivo.

\section{Tabla 7}

Medidas de tendencia central estimadas

\begin{tabular}{cc}
\hline Media & $\$ 6.471,490$ \\
Desv. Est & $\$ 3.531,214$ \\
No. de datos & 5 \\
\hline
\end{tabular}

Fuente: Elaboración propia. 
Tabla 8

Intervalo de confianza estimado.

\begin{tabular}{l}
\hline cii 56471.4903531 .214 \\
Variable | Obs Mean Std. Err. [95\% Conf. Interval] \\
| 56471.491579 .2072086 .90910856 .07 \\
\hline
\end{tabular}

Fuente: Elaboración propia

A partir de los datos estimados, se concluye que la DAP media para la zona de estudio por la afectación del proyecto de aprovechamiento hidroeléctrico puede ascender a COP $\$ 6.471$ pesos mensuales por hogar, y se puede mantener en un rango de $\$ 2.086$ y $\$ 10.856$ pesos colombianos de 2014 .

Para corroborar el error de estimación se aplica el test de viabilidad con los siguientes resultados:

Tabla 9

Error de estimación de la transferencia.
La tabla 9 permite observar los valores estimados de error de transferencia para cada uno de los estudios seleccionados, donde los valores negativos expresan subestimación del valor de DAP estimado por el estudio y los valores positivos expresan sobreestimación. Se asume un error promedio de transferencia del $35 \%$, inferior a los encontrados en la literatura y se generan errores en un rango de entre el $-57 \%$ y el $126 \%$.

\section{Conclusiones}

La transferencia de beneficios es una metodología adecuada para aproximar medidas de bienestar cuando es complicado o se dificulta por alguna razón recolectar información primaria. La aplicación de

\begin{tabular}{lccc}
\hline \multicolumn{1}{c}{ Estudio } & $\begin{array}{c}\text { Error de estimación } \\
\text { (DAP media estimada) }\end{array}$ & $\begin{array}{c}\text { Error de estimación } \\
\text { (DAP B-) }\end{array}$ & $\begin{array}{c}\text { Error de estimación } \\
\text { (DAP B+) }\end{array}$ \\
\hline Correa, Osorio y & $-43 \%$ & $-82 \%$ & $-4 \%$ \\
Mendieta (2010) & $-15 \%$ & $-72 \%$ & $43 \%$ \\
Ponce et al (2011) & $161 \%$ & $-16 \%$ & $337 \%$ \\
Gunawardena (2010) & $-13 \%$ & $-72 \%$ & $46 \%$ \\
Han, Kwak y Yoo (2008) & $82 \%$ & $-41 \%$ & $206 \%$ \\
Tao, Yan y Zhan (2012) & $\underline{35 \%}$ & $\underline{-57 \%}$ & $\underline{126 \%}$ \\
Error promedio estimado & & & \\
\hline
\end{tabular}


los distintos métodos de transferencia de beneficios requiere de un proceso riguroso de revisión bibliográfica para escoger los estudios con sitios de características similares al sitio de intervención. El método de transferencia de beneficios a utilizar depende, en gran parte, de la información existente, ya que la literatura económica no provee evidencia empírica contundente a favor o en contra de ningún método.

Estos valores económicos del impacto en bosque son más confiables cuando se obtienen a partir de la percepción de los hogares, a través de su disposición a pagar por conservación o pérdida de bienestar. Esto último se logra por medio de la aplicación de métodos de preferencias declaradas o de preferencias reveladas. Ahora bien, en presencia de limitaciones de tiempo y de disponibilidad de recursos, las agencias gubernamentales pueden tomar decisiones de política pública, con relación a priorización de recursos, a partir de la realización de análisis costo-beneficio basados en el método de transferencia de beneficios.

Finalmente, es importante establecer las limitaciones del ejercicio de valoración desarrollado en este estudio. En este sentido, se plantean cinco aspectos críticos, a saber: localización geográfica, tipo de estudio, activo ambiental, técnica de valoración y medida económica. Con relación a la localización geográfica, la limitación del ejercicio radica en que los estudios empleados se desarrollaron en países europeos con condiciones socioeconómicas, culturales y ambientales diferentes a las colombianas. No obstante, con la idea de reducir este sesgo se transformaron los valores de transferencia a valores de paridad de poder adquisitivo, con el fin de realizar una transferencia lineal de los datos y poder obtener valores DAP por impacto en la cobertura boscosa ajustados a los ingresos promedio en Colombia.

\section{Referencias}

Adger, W., Brown, K., Cervigni, R., y Moran, D. (1995). Total Economic Value of Forest in Mexico. Ambio, 24(5): 286296.

AEVAL. (2013). CAF. Recuperado de http://www.eipa.eu/files/File/CAF/ ES_CAF_2013.pdf

ANLA. (2006). Diagnóstico Ambiental de Alternativas (DAA). Recuperado de http://www.anla.gov.co/diagnosticoambiental-alternativas

ANLA. (2006). Estudios de Impacto Ambiental (EIA). Recuperado de http:// www.anla.gov.co/documentos/normativa/tdr_materiales_construccion.pdf 
Azqueta, D. (1994). Valoración económica de la calidad ambiental. Madrid: Mcgraw-Hill.

Bennett, J. (2011). The international handbook on Non-market Environmental Valuation. Cheltenham: Edward Elgar.

Brouwer, R. y Spaninks, F. (1999). The validity of environmental benefits transfer: Further empirical testing. Environmental and Resource Economics, 14(1): 95-117

Brouwer, R. (2000). Environmental value transfer: State of the art and future prospects. Ecological Economics, 32(1): 137-152

Desvousges, W., Johnson, F., Dunford, R., Hudson, S., Wilson, K., Boyle, K. (1992). Measuring Nonuse Damages Using Contingent Valuation: An Experimental Evaluation of Accuracy. Recuperado de http://www. rti.org/sites/default/files/resources/ bk-0001-1009_web.pdf doi:10.3768/ rtipress.2009.bk.0001.1009

Figueroa, E. (2010). Valoración económica detallada de las áreas protegidas de Chile. En Fondo del Medio Ambiente Mundial, Ministerio del Medio Ambiente (Gobierno de Chile) y Programa de las Naciones Unidas para el Desarrollo (eds.), Proyecto GEFMMA-PNUD. Creación de un sistema integral de áreas protegidas para Chile. (107-280). Chile: Salesianos.

Guo, Z., Li, Y., Xiao, X., Zhang, L. y Gan, Y. (2007). Hydroelectricity Production and Forest Conservation in Watersheds. Ecological Applications, 17(6): 1557-1562.

Hoffmann, R. (1996). Problemas y perspectivas de la valoración de recursos y procesos naturales: Análisis de costo-beneficio en áreas rurales del "Tercer Mundo". Economía Informa, 253:29-44.

Loomis, J. (1992). The evolution of a more rigorous approach to benefit transfer: Benefit function transfer. Water Resources Research, 28(3): 701-705.

Macías, D. (2010). Las áreas protegidas privadas: una estrategia para el desarrollo sustentable. Recuperado de http://cmsdata.iucn.org/downloads/ cel10_mendez.pdf

Mendieta. J. (1999). Manual de valoración económica de bienes no mercadeables: aplicaciones de las técnicas de valoración no mercadeables y el análisis costo beneficio y medio ambiente. Bogotá: Universidad de los Andes. 
Ministerio de Ambiente, Vivienda y Desarrollo Territorial. MAVDT. (2010). Evaluación económica de impactos ambientales en proyectos sujetos a licenciamiento ambiental. [En línea]. Recuperado de http://www.ucipfg.com/ Repositorio/MAES/MAES-09/Unidades_academicas/Unidad_5/Manualtecnico.pdf

Navrud, S. y Bergland, O. (2001). Value Transfer and Environmental Policy. Policy Research Brief No. 8. Cambridge Research for the Environment. [En línea]. Recuperado de http://www.clivespash.org/eve/prb8-edu.pdf

Navrud, S. y Ready, R. (2007). Environmental values transfer: Issues and Methods. Serie The economics of non-markets goods and resources. Springer.

Parsons, G. y Kealy, M. (1994). Benefits transfer in a random utility model of recreation. Water Resources Research, 30(8): 2477-2484.

Pearce, D. y Turner, R. (1990). Economic of natural resourse and the enviroment. New York: Harvester Wheatsheaf.
República de Colombia. Ministerio de Minas y Energía. (2012). Minminas. Recuperado de https://www.minminas. gov.co/inicio

Rosenberger, R. y Loomis, J. (2000). Benefit Transfer of Outdoor Recreation Use Values: A Technical Document Supporting the Forest Service Strategic Plan (2000 Revision). EEUU: Departamento de Agricultura de los Estados Unidos.

USDA. (2001). General Technical Report RMRS-GTR-72. [En línea]. Recuperado de https://www.fs.fed.us/rm/pubs/ rmrs_gtr072.pdf

Vásquez, F., Cerda, S. y Orrego (2007). Valoración Económica del Medio Ambiente. Buenos Aires: Thomson Internacional.

Walsh, R., Johnson, D. y Mckean, J. (1992). Benefit transfer of Outdoor Recreation Demand Studies 1968-1988. Water Resourseces Research, 28(3): 707-713.

Woodward, R. y Wui, Y-S. (2001). The economic value of wetland service: a metaanalysis. Ecological Economics, 2(37): $257-270$. 